\section{Pollen Exine Formation}

THE chief purpose of Mepham and Lane in their recent article $^{1}$ seems to be to establish that the exine of the pollen grain is formed by secretion from within the microspore and owes little or nothing to the activity of the disorganizing tapetum. Their interesting new observations and their substantial claims to reinterpretation of the ontogeny of the pollen grain suffer from overcondensation, and it is a pity that they had no space to consider other recent electron microscopo studies, at close time intervals, of pollen grain development from the pollen-mother cell stage to pollen maturity ${ }^{2-6}$. They claim that in Tradescantia bracteata "the bulk of the exine is produced at an early stage". Heslop-Harrison's pioneer study of Silene pendula ${ }^{2}$, however, made it clear that, although the pattern of the exine is clearly determined while the microspores are within their own callose envelopes and the common callose envelopes of the pollen mother cell, the most important part of exine development occurs after dissolution of the callose walls and exposure of the exine to the fluid of the anther loculus. A similar conclusion has emerged from studies of Helleborus foetidus and Endymion non-scriptus made in the Botany School, Cambridge $e^{3-6}$.

There have been claims that the sporopollenin of the developing exine is pre-synthesized in the tapetum and transferred in particulate form to the exine, but I, like Mepham and Lane, have found no evidence for this. On the other hand, this in no way precludes the possibility that exine precursors in solution move from the disorganizing tapetum to the microspores; they may then condense directly on the growing exine surfaces or it may be necessary for them first to enter the microspore protoplast for subsequent secretion through the cell membrane. Although Ubisch bodies are apparently not formed in Tradescantia bracteata, in other species which do have them the deposition of sporopollenin takes place on the surfaces of spherical, probably lipid, bodies that are formed in the tapetal cells and have emerged through the tapetal cell membranes. Not until they are thus brought into contact with the anther loculus is sporopollenin deposited on them, possibly under the control of a surface membrane that originates from the endoplasmic reticulum from which the pro-Ubisch bodies seem to originate. Here at least the formation of sporopollenin is independent of secretion from the microspore protoplast. This phenomenon, together with many reports of membrane structures in both early and late stages of exine development, leads one to conjecture that such membranes may well be the seat of sporopollenin condensation, whether in Ubisch bodies or in primary or secondary pollen grain exine, in both of which the process proceeds at the same time.

Mepham and Lane seem to associate exine thickening with processes involving the intine which, in our experience, forms only when exine development is almost complete.

The electron micrographs which they now publish, although highly interesting, require much support and explanation. The exine elements in them are unlabelled so that one can only infer the authors' interpretation. This is made harder by the fact that the dark-stained laminate material in Fig. 1 is regarded as endexine (that is, sporopollenin), whereas similarly stained laminate material in Fig. 2 is regarded as "lipid exudate" with the ektexine (sporopollenin) now scarcely stained at all. It seems possible that Fig. 2 simply shows a tangential (not radial) section through the exine at a higher or lower level than in Fig. 1, but with similar staining of the wall elements. If this is so we are concerned with a lamellate structure of the exine such as seems to be characteristic of secondary exine in many species of plants, notably Ipomoea coerulea ${ }^{7}$; in this case, it may be important that the electron lucent bands shown here in Tradescantia are of similar width (40 $\AA$ ) to the "white lines" often seen in sections of secondary exine ${ }^{10}$.
If, alternatively, Mepham and Lane's own interpretation of Fig. 2 is followed, it would seem that the dark reticular pattern seen in section tangential to the surface of the grain is that of the interbacular cavities of the exine, now filled with a lipid deposit, possibly derived from the periplasmodium filling the anther sac. This is a situation like that described by Chambers and myself for Tilia platyphyllos ${ }^{8}$, and one that is undoubtedly very widespread. The pattern of parallel electron lucent bands traversing the lipid filling of the exine cavities recalls that described by Dunbar in the exine of Plumbago capensis ${ }^{9}$. What is altogether new is the interpretation of Mepham and Lane that this filling is derived not from the periplasmodium, but by direct secretion of lipids by the microspore protoplast, through the cell membrane, intine and endexine. It seems perfectly possible that this may operate, but there is a great deal of evidence, old and new, that the pigmented contents of the anther loculus come to be very closely associated with the pollen-exine, where indeed they have been given numerous conjectural functions. It would be worth looking for evidence as to whether the pattern of parallel electron lucent bands in the exine cavity might not be a derivative of the early lamellar structure of the primexine, for a fibrillar texture of similar orientation persists in several species through the middle stages of exine development.

There is scarcely enough evidence in their highly condensed account to allow that Mepham and Lane's results "conflict with the widely held idea that tapetal breakdown is purely a degenerative process". Likewise the notion that the periplasmodium within the anther sac is not merely a substrate reserve for the developing pollen grains, but has inherent organizing potentialities of its own, seems to call for more substantial evidence than they have presented so far.

\section{H. Godwin}

Botany School,

University of Cambridge.

Received September 5, 1968.

${ }^{1}$ Mepham, R. H., and Lane, G. R., Nature, 219, 961 (1968).

2 Heslop-Harrison, J., Grana Palynol., 4, 7 (1963).

Echlin, P., Chapman, B., Godwin, H., and Angold, R., Proc. Sixth Intern. Cong. Electron Microsc., Kyoto, Electron Microsc., 2, 315 (1966).

4 Echlin, P., and Godwin, H., J. Cell Sci., 3, 161 (1968).

${ }^{5}$ Echlin, P., and Godwin, H., J. Cell Sci., 3, 175 (1968).

${ }^{6}$ Angold, R. F., Rev. Palaeobot. Palynol., 3, 205 (1968).

?Godwin, H., Echlin, P., and Chapman, B., Rev. Palaeobot. Palynol., 3, $181(1967)$.

${ }^{8}$ Chambers, T. C., and Godwin, H., New Phytol, 80, 393 (1961),

- Dunbar, A., Grana Palynol., y, 10 (1967).

${ }^{10}$ Godwin, II., New Phytol., 67, 667 (1968).

\section{Relative Mobility of some Organic Nitrogenous Compounds in the Xylem of Apple Shoots}

In the apple, as in many other perennial plants, all the absorbed nitrogen is usually reduced and combined into amino-acids in the youngest roots and is subsequently translocated in the form of such compounds. Xylem sap, which has been extracted from the terminal shoots of mature trees by a vacuum technique, contains nitrogen, largely as aspartic acid and asparagine, but also as arginine, glutamic acid and glutamine. There may be traces of a number of other amino-acids, depending on season and on added fertilizer ${ }^{1,2}$.

To investigate the relative mobilities of these com. pounds a technique has been developed for perfusing standard lengths of $1 \mathrm{yr}$ old apple shoots, approximately $12 \mathrm{~cm}$ long and $0.5 \mathrm{~cm}$ in diameter. A ring of bark was cut away from each end of the shoot and short lengths of rubber tubing were attached directly to the exposed wood. 